Artículo original

\title{
Validación del puntaje de valoración de la gravedad de la enfermedad pulmonar obstructiva crónica en población colombiana en un servicio de atención primaria
}

\author{
Jorge Mario Estrada-Álvarez¹, Juan Pablo Orozco-Hernández¹,2, Luis Evelio \\ Aristizábal-Franco ${ }^{3}$ \\ ${ }^{1}$ Grupo de Investigación en Salud Comfamiliar, Clínica Comfamiliar, Pereira, Colombia \\ ${ }^{2}$ Programa de Medicina, Universidad Tecnológica de Pereira, Pereira, Colombia \\ ${ }^{3}$ Grupo de Investigación en Gerencia del Cuidado, Universidad Libre de Pereira, Pereira, Colombia
}

Introducción. La enfermedad pulmonar obstructiva crónica (EPOC) es un importante problema de salud mundial con una alta morbimortalidad. Se requiere la medición de la gravedad de la enfermedad mediante una herramienta de fácil aplicación, bajo costo y fácil disponibilidad en áreas rurales.

Objetivo. Evaluar la validez y confiabilidad del puntaje de valoración de la gravedad de la EPOC (Chronic Obstructive Pulmonary Disease Severity Score, COPDSS) en una población de atención primaria en Colombia.

Materiales y métodos. Se hizo un estudio de corte transversal en una muestra de 100 pacientes con diagnóstico de EPOC según las guías GOLD. La validez concurrente se evaluó correlacionando los resultados del COPDSS con otras variables de importancia como las del cuestionario sobre la enfermedad respiratoria crónica (Chronic Respiratory Disease Questionnaire, CRQ) y el volumen espiratorio forzado en el primer segundo (Forced Expiratory Volume in one second, $\mathrm{FEV}_{1}$ ).

Resultados. EI COPDSS presentó una correlación significativa con el $\mathrm{FEV}_{1}(r=-0,33)$, con el CRQ $(r=-0,57)$ y sus dimensiones: disnea $(r=0,51)$, fatiga $(r=0,53)$, función emocional $(r=0,43)$ y control de la enfermedad $(r=0,50)$. En el análisis factorial se determinó un solo factor con una varianza acumulada de 59,1\%. El análisis de coherencia interna mostró un alfa de Cronbach de 0,76, valor este considerado adecuado.

Conclusiones. Se observó que el uso del COPDSS como cuestionario de valoración de la gravedad de pacientes con EPOC en Colombia tenía validez y confiabilidad adecuadas y

Recibido: 05/08/2019

Aceptado: $17 / 06 / 2020$

Publicado: 23-06/2020

Citación:

Estrada-Álvarez JM, Orozco-Hernández JP, Aristizábal-Franco LE. Validación del puntaje de valoración de la gravedad de la enfermedad pulmonar obstructiva crónica en población colombiana en un servicio de atención primaria. Biomédica. 2020;40:664-72.

https://doi.org/10.7705/biomedica.5123

\section{Correspondencia:}

Juan Pablo Orozco-Hernández, Clínica Comfamiliar,

Avenida Circunvalar $N^{\circ}$ 3-01, Pereira, Colombia Teléfono: (57) (320) 6067516

jporozco1994@ hotmail.com

Contribución de los autores:

Jorge Mario Estrada-Álvarez: análisis estadísticos Juan Pablo Orozco-Hernández: análisis estadístico y redacción del manuscrito

Luis Evelio Aristizábal-Franco: recolección de datos Todos los autores participaron en el planteamiento de la idea y el desarrollo del estudio, el análisis crítico y la aprobación del manuscrito.

Financiación:

El trabajo fue financiado por los propios autores.

Conflicto de intereses:

Ninguno que es de fácil aplicación en la atención primaria.

Palabras clave: enfermedad pulmonar obstructiva crónica; índice de gravedad de la enfermedad; atención primaria de salud; espirometría; encuestas y cuestionarios; Colombia.

Validation of chronic obstructive pulmonary disease severity score (COPDSS) for primary care in a Colombian population

Introduction: Chronic obstructive pulmonary disease (COPD) is a major global health problem causing high morbidity and mortality. Easy to apply, cost-effective, and available COPD severity measures are required in rural areas.

Objective: To assess the validity and reliability of the COPD Severity Score (COPDSS) in Colombian patients attended in primary care.

Materials and methods: A cross-sectional study was conducted in a sample of 100 patients diagnosed with COPD according to GOLD guidelines. Convergent validity was assessed by correlating the COPDSS results with other important variables such as those assessed through the Chronic Respiratory Disease Questionnaire (CRQ) and the forced expiratory volume in one second $\left(\mathrm{FEV}_{1}\right)$.

Results: The COPDSS showed a significant correlation with the $\mathrm{FEV}_{1}(\mathrm{r}=-0.33)$, with the CRQ ( $r=-0.57)$ and its dimensions: Dyspnea $(r=0.51)$, fatigue $(r=0.53)$, emotional function $(r=0.43)$, and disease control $(r=0.50)$. The factor analysis identified a single factor with a cumulative variance of $59.1 \%$. The internal consistency analysis showed an adequate Cronbach's alpha of 0.76 .

Conclusions: The use of the COPDSS as an assessment test for COPD patients in Colombia showed adequate validity, reliability, and easy application in primary care.

Keywords: Pulmonary disease; chronic obstructive; severity of illness index; primary health care; spirometry; surveys and questionnaires; Colombia. 
La enfermedad pulmonar obstructiva crónica (EPOC) es un importante problema de salud mundial con una alta morbimortalidad (1). Actualmente, la EPOC es la tercera causa de muerte en el mundo, ocupa el quinto lugar en términos de carga de la enfermedad, tiene un riesgo acumulado de por vida estimado del $25 \%$, y afecta a hombres y mujeres por igual (2). En Colombia, se estimó una prevalencia del 8,9\% en cinco ciudades para el 2007 (3) y una importante carga de la enfermedad, con 208.166 años perdidos de vida ajustados por discapacidad para el 2015, ocupando el tercer lugar en Latinoamérica después de México y Argentina (1).

En general, las tasas de mortalidad por EPOC específicas para la edad están disminuyendo, y el aumento global en el número de muertes se relaciona con el crecimiento y el envejecimiento de la población, ya que la enfermedad afecta predominantemente a los ancianos (1). En los países de altos ingresos, el tabaquismo es el principal factor de riesgo para el desarrollo de la EPOC, pero también se reconocen otros factores entre los que se destacan la exposición al humo de la biomasa, las infecciones en la niñez, los factores genéticos y el asma alérgica, especialmente en los países de ingresos bajos y medios como Colombia, Argentina, China y Brasil, entre otros (4). Aunque se han logrado avances en el tratamiento de la EPOC, es necesario encontrar terapias que reduzcan la progresión de la enfermedad y la mortalidad (5).

Actualmente, hay diversos métodos para clasificar la gravedad de los pacientes con EPOC con fines de pronóstico y tratamiento, recurriendo, entre otros aspectos, a la valoración de la función pulmonar alterada, la disnea grave, el tabaquismo activo y el puntaje de gravedad de la EPOC (Chronic Obstructive Pulmonary Disease Severity Score, COPDSS) (6).

EI COPDSS es un cuestionario corto y de fácil aplicación desarrollado por Eisner, et al. (6), ya traducido y validado en español (7). El COPDSS ha demostrado tener una buena validez y capacidad predictiva para exacerbaciones por la EPOC, con mejores resultados predictivos que otras variables (8). La clasificación de la gravedad y del riesgo de los pacientes con un método fácil y práctico es de relevancia para la práctica clínica, ya que la disponibilidad de la espirometría puede ser limitada en áreas de difícil acceso o en zonas rurales. Asimismo, la implementación de un método de bajo costo y mayor accesibilidad permitiría un mejor manejo de la EPOC, con intervenciones más tempranas y pertinentes, reduciendo la morbilidad, la mortalidad y los costos asociados con la atención en salud de estos pacientes a largo plazo.

En Colombia, las guías de práctica clínica no incluyen métodos de clasificación de la gravedad de fácil y rápida implementación, y el COPDSS no ha sido validado en el país. Asimismo, en los países de bajos y medianos ingresos, la gravedad y los factores de riesgo asociados con la EPOC pueden ser diferentes a los de los países de mayores ingresos.

Dada la necesidad de un método de clasificación de la gravedad de la EPOC de fácil aplicación y acceso, el objetivo de este estudio fue validar el COPDSS en una población de Colombia con diagnóstico de EPOC confirmado por espirometría.

\section{Materiales y métodos}

\section{Pacientes y muestra}

Se desarrolló un estudio de corte transversal con enfoque de validación de instrumentos basado en lo sugerido por diferentes autores (9-11). Se 
seleccionaron 100 pacientes pertenecientes a un programa de EPOC, todos con confirmación del diagnóstico mediante espirometría según las guías GOLD (12).

Los criterios de inclusión fueron: una edad igual o mayor de 40 años; diagnóstico de EPOC según los criterios de la guía GOLD; índice $\mathrm{FEV}_{1}$ / $\mathrm{FVC}<0,7$ en la espirometría posterior a la broncodilatación, y presencia de alguno de los siguientes factores de riesgo: exposición a humo de tabaco, de leña, o exposición ocupacional respiratoria a partículas, además de síntomas respiratorios crónicos (disnea, tos o expectoración).

Se excluyeron aquellos pacientes con algún déficit cognitivo que impidiera la respuesta adecuada del cuestionario y aquellos con una espirometría que no cumplía con los criterios de buena calidad.

El tamaño de la muestra se definió siguiendo la recomendación de Streiner, et al. (11), es decir, elegir entre 5 y 7 individuos por ítem del cuestionario y garantizar una muestra no menor de 100 individuos.

El estudio fue aprobado por el Comité de Bioética del Hospital de Santa Mónica (Dosquebradas, Risaralda) como un estudio "sin riesgo" según la Resolución 8430 de 1993 expedida por el Ministerio de Salud de Colombia y acorde con la Declaración de Helsinki. Todos los pacientes firmaron el consentimiento informado aceptando participar en la investigación.

\section{Recolección de información}

Siguiendo los procedimientos de validación propuestos para este tipo de cuestionarios, se hizo la traducción al español. Aunque ya se había validado este cuestionario en España (7), debido a las diferencias lingüísticas con el español de Colombia y a las de algunos medicamentos empleados en el país, fue necesaria una traducción ajustada al contexto nacional (11). En primer lugar, la versión original del cuestionario fue traducida por un profesional bilingüe experto en el área específica, versión que fue revisada después por otro profesional bilingüe para reducir el riesgo de errores de traducción.

El cuestionario consta de cinco aspectos generales por evaluar: síntomas respiratorios, con un máximo de 7 puntos; uso de corticoides, con un máximo de 5 puntos; uso de otros medicamentos, con un máximo de 10 puntos, y hospitalización o uso de oxígeno domiciliario, con un máximo de 13 puntos, para un puntaje total en un rango entre 0 y 35 puntos en el que los puntajes más altos se asocian con una mayor gravedad de la EPOC. Cada ítem tiene un peso a priori basado en aspectos clínicos de la enfermedad y su contribución esperada en la gravedad general de la EPOC (6).

Cada paciente fue citado a una consulta clínica para valoración que incluyó una entrevista cara a cara utilizando el cuestionario y preguntas sobre los datos sociodemográficos y aspectos clínicos de la enfermedad. La información fue recolectada por profesionales en terapia respiratoria entrenadas previamente en la aplicación de los cuestionarios y en la toma de espirometrías. La espirometría se ajustó a las guías de la American Thoracic Society (ATS) y la European Respiratory Society (ERS) (13).

\section{Validación}

Como parte del proceso de validación concurrente, se plantearon diferentes hipótesis de relación que permitieran dar cuenta de la variable latente de gravedad de la enfermedad, para lo cual se consideraron como parámetros 
clínicos la espirometría y la calidad de vida relacionada con la salud. Esta última se midió mediante el Chronic Respiratory Questionnaire (CRQ) desarrollado por Guyatt, et al. (14), y validado en Colombia previamente (15). Este cuestionario también fue administrado por profesionales en terapia respiratoria previamente entrenadas en su diligenciamiento.

El CRQ se compone de 20 preguntas o ítems y se divide en cuatro dimensiones de la calidad de vida relacionada con la salud: disnea, fatiga, función emocional y control de la enfermedad. El paciente selecciona una respuesta para cada ítem a partir de una escala de siete posibles respuestas equidistantes. Se puntúan por separado cada una de las dimensiones y también se obtiene una puntuación total. En el CRQ las puntuaciones más altas indican una mejor calidad de vida.

\section{Análisis estadístico}

Se hizo un análisis estadístico descriptivo con medidas de tendencia central expresadas como medias y medianas, y las medidas de dispersión, como varianza, desviación estándar y percentiles. Para la evaluación de la validez del constructo, se hizo un análisis factorial exploratorio con extracción por el método de componentes principales usando como criterio la selección de factores con valores propios mayores de 1; la rotación de los factores extraídos se hizo mediante el método varimax con normalización de Kaiser.

En todos los análisis se cumplieron previamente los supuestos para la aplicación del método, es decir, una prueba de esfericidad de Barttlet significativa y la medida de adecuación de Kaiser-Meyer-Olkin.

La correlación bivariada entre el puntaje del cuestionario de gravedad y las otras medidas clínicas, se hizo mediante los coeficientes de correlación de Spearman y de correlación parcial. La coherencia interna (confiabilidad) se estimó con el alfa de Cronbach. En todos los análisis se utilizó un nivel de significación de 0,05 . Se utilizó el programa Stata $14.0^{\mathrm{TM}}$ para los análisis estadísticos.

\section{Resultados}

\section{Características clínicas de los pacientes}

Se incluyeron 106 pacientes para la validación del COPDSS, de los cuales se excluyeron seis debido a espirometrías que no cumplían con los criterios de calidad, es decir, el análisis se hizo en 100 pacientes.

La edad promedio fue de 73,4 $\pm 9,2$ años y el $52 \%$ pertenecía al sexo femenino. La distribución por estado civil fue la siguiente: solteros, $32 \%$, casados, $23 \%$ y viudos, $24 \%$.

Los parámetros espirométricos mostraron un promedio de porcentaje predicho en el $\mathrm{VEF}_{1}$ (volumen espiratorio forzado al primer segundo) de 59,3\% y de $68,4 \%$ en la capacidad vital forzada.

En cuanto al CRQ, los valores cercanos a siete evidenciaban la mejor calidad de vida y, aquellos cercanos a uno, la peor. En todas las dimensiones se obtuvo un puntaje promedio por encima de cinco, lo que indicaba una buena calidad de vida entre los evaluados. Otras características clínicas de los pacientes se pueden ver en el cuadro 1. 


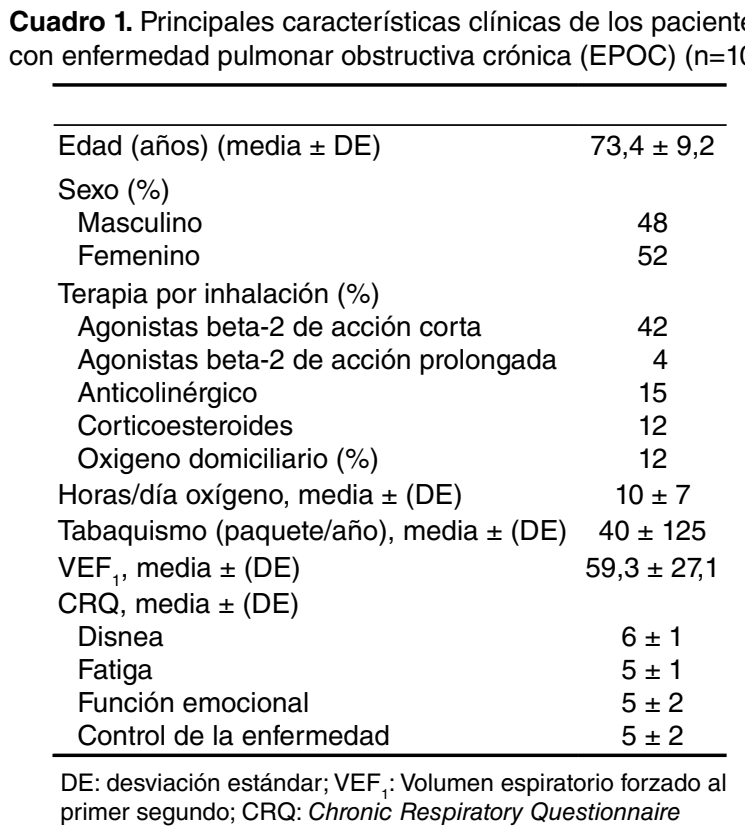

En cuanto a los resultados del COPDS, el puntaje objetivo mostró una distribución asimétrica (prueba de Kolmorogov, $p<0,0001$ ), con una mediana de 7 (rango intercuartílico, $\mathrm{RIC}=4-12$ ). La proporción de respuestas para cada ítem evaluado se presenta en el cuadro 2.

\section{Validez del constructo}

En la evaluación de esta forma de validez, el análisis factorial dio como resultado una matriz de correlaciones significativas según la prueba de Bartlett (ji al cuadrado $=126,53 ; p=0,0001$ ), con un coeficiente de adecuación de de Kaiser-Meyer-Olkin de 0,7, lo que indicó que el método de análisis factorial podía aplicarse a los datos del estudio.

En la determinación de la estructura interna de la escala, que permite el establecimiento de la variable latente (en este caso, la gravedad de la enfermedad), se encontró un solo factor con valor propio de 2,36 y una proporción de varianza acumulada explicada por este factor de 59,1\%. Las cargas factoriales, que indican el peso que tiene cada área sobre el factor (gravedad de la enfermedad) de cada una de las áreas de evaluación en el cuestionario, se muestran en el cuadro 3.

\section{Validez concurrente: correlación con otros parámetros}

En el análisis de asociación con parámetros de la función pulmonar, el VEF ${ }_{1}$ tuvo una correlación significativa con el puntaje de gravedad del cuestionario $(r=-0,33 ; p=0,0008)$ después de controlar por la edad. En el cuadro 4 se presentan las correlaciones entre la calidad de vida relacionada con la salud y las dimensiones de la misma según el COPDSS, controlando por los valores del porcentaje predicho del $\mathrm{VEF}_{1}$ y la edad. Se presentaron correlaciones moderadas con las dimensiones de disnea y de fatiga, y débiles con las de función emocional y control de la enfermedad.

\section{Confiabilidad (coherencia interna)}

La correlación entre los ítems de la escala, la cual muestra la coherencia interna del cuestionario, dio como resultado un alfa de Cronbach de 0,76, que corresponde a una confiabilidad adecuada. 
Cuadro 2. Ítems en el cuestionario de gravedad de la enfermedad pulmonar obstructiva crónica (EPOC)

\begin{tabular}{|c|c|c|}
\hline Ítem & Puntaje & $\%$ \\
\hline \multicolumn{3}{|l|}{ Síntomas respiratorios (máximo, 7 puntos) } \\
\hline Ninguno & 0 & 21 \\
\hline Caminando de prisa o apurado en terreno plano & 1 & 25 \\
\hline $\begin{array}{l}\text { Caminando con otra persona de su misma edad en } \\
\text { terreno plano }\end{array}$ & 2 & 0 \\
\hline $\begin{array}{l}\text { Tiene que detenerse para tomar aire cuando camina a } \\
\text { su ritmo en un nivel plano }\end{array}$ & 3 & 54 \\
\hline \multicolumn{3}{|l|}{ Disnea en los pasados 14 días o noches } \\
\hline Ninguna & 0 & 34 \\
\hline 1 a 2 días o noches & 1 & 14 \\
\hline 3 a 6 días o noches & 2 & 26 \\
\hline 7 a 13 días o noches & 3 & 15 \\
\hline Todos los días o noches & 4 & 11 \\
\hline \multicolumn{3}{|l|}{ Uso de corticoide sistémico (máximo, 5 puntos) } \\
\hline Alguna vez lo ha usado & 1 & 18 \\
\hline Usado en el pasado año & 3 & 17 \\
\hline Usado en las pasadas dos semanas & 1 & 20 \\
\hline \multicolumn{3}{|l|}{$\begin{array}{l}\text { Uso de otra medicación (máximo, } 10 \text { puntos) } \\
\text { Inhaladores en las pasadas dos semanas }\end{array}$} \\
\hline Agonistas beta 2 de corta acción & 1 & 38 \\
\hline Agonistas beta 2 de acción prolongada & 1 & 7 \\
\hline Corticoide inhalado & 1 & 16 \\
\hline Anticolinérgicos & 1 & 25 \\
\hline \multicolumn{3}{|l|}{ Uso de nebulizador en las pasadas dos semanas } \\
\hline Agonista beta 2 de acción corta & 1 & 6 \\
\hline Anticolinérgicos & 1 & 9 \\
\hline \multicolumn{3}{|l|}{ Medicación oral } \\
\hline Teofilina en las pasadas dos semanas & 1 & 5 \\
\hline Agonistas beta 2 & 1 & 1 \\
\hline \multicolumn{3}{|l|}{$\begin{array}{l}\text { Antibiótico para condiciones pulmonares } \\
\text { en los pasados } 12 \text { meses }\end{array}$} \\
\hline Uno a dos periodos & 1 & 13 \\
\hline Tres o más periodos & 2 & 87 \\
\hline \multicolumn{3}{|l|}{$\begin{array}{l}\text { Hospitalización, intubación, uso de oxígeno } \\
\text { domiciliario (máximo, } 13 \text { puntos) }\end{array}$} \\
\hline Hospitalizado por EPOC en los pasados cinco años & 3 & 39 \\
\hline Intubado por EPOC en los pasados cinco años & 5 & 1 \\
\hline Oxigeno domiciliario actualmente & 5 & 11 \\
\hline
\end{tabular}

Cuadro 3. Cargas factoriales de cada área de evaluación de la escala de gravedad de la enfermedad pulmonar obstructiva crónica (EPOC)

\begin{tabular}{lc}
\hline Áreas & Factor 1 \\
\hline Síntomas respiratorios & 0,59 \\
Uso de medicamentos & 0,89 \\
Hospitalización, intubación, oxígeno & 0,84 \\
Uso corticoide sistémico & 0,69 \\
\hline
\end{tabular}

Cuadro 4. Correlación entre los puntajes de la calidad de vida relacionada con la salud (CRQ) y escala de gravedad de la enfermedad (COPDSS)

\begin{tabular}{lcc}
\hline Calidad de vida relacionada con la salud & $\begin{array}{c}\text { Coeficiente correlación } \\
\text { no ajustado }\end{array}$ & $\begin{array}{c}\text { Coeficiente de } \\
\text { correlación ajustado* }\end{array}$ \\
\hline Puntaje total en el CRQ & $-0,48$ & $-0,57$ \\
Disnea & $-0,49$ & $-0,51$ \\
Fatiga & $-0,50$ & $-0,53$ \\
Función emocional & $-0,37$ & $-0,46$ \\
Control de la enfermedad & $-0,38$ & $-0,50$ \\
\hline
\end{tabular}

*Valor del coeficiente de correlación parcial ajustado por VEF y edad. Todos los análisis fueron estadísticamente significativos $(p<0,001)$. 


\section{Discusión}

La EPOC es una de las principales causas de mortalidad y morbilidad a nivel mundial, con una alta carga de la enfermedad y costos para el sistema de salud (1). En Colombia, se han hecho algunos estudios en esta población $(3,15-19)$, pero hasta el momento no se había validado una escala de gravedad de la EPOC, siendo esta la primera validación en el país. Este estudio en una población de pacientes con EPOC confirmada por espirometría demuestra que el uso del COPDSS es factible, de fácil aplicación en los servicios de atención primaria, con una adecuada correlación con la calidad de vida y la función pulmonar, y con una coherencia interna apropiada.

Actualmente, la estratificación de la gravedad de la EPOC se hace con base en parámetros fisiológicos, como la función pulmonar $\left(\mathrm{FEV}_{1}\right)$ según las guías GOLD (12), y en los síntomas de disnea y de exacerbación (20) que, aunque son medidas de gravedad de la enfermedad, no tienen en cuenta resultados de relevancia como la calidad de vida relacionada con la salud y la discapacidad. De ahí la relevancia de la adopción de nuevas formas de medición de la gravedad de la enfermedad con base en múltiples dimensiones y aspectos, como el uso de inhaladores, la necesidad de oxígeno domiciliario, las exacerbaciones que conlleven la hospitalización en unidades de cuidados intensivos y la reducción de síntomas.

Asimismo, otras medidas utilizadas en estudios clínicos, como la caminata de seis minutos (21) y el índice BODE (22), entre otros (20), deben aplicarse en un ámbito clínico, lo cual limita su uso en el marco de encuestas poblacionales epidemiológicas. A esto se suma que la determinación de la gravedad mediante la espirometría puede ser costosa y no estar disponible en zonas de difícil acceso o en áreas rurales. Se ha establecido que, a diferencia de quienes viven en zonas urbanas, en pacientes con EPOC de zonas rurales la mortalidad y la prevalencia de EPOC son mayores $(23,24)$, y que las fallas en el diagnóstico conllevan un tratamiento menos efectivo (25).

En este estudio, el COPDSS demostró tener validez concurrente, pues hubo correlaciones significativas con parámetros como la calidad de vida y la función pulmonar $\left(\mathrm{VEF}_{1}\right)$ de igual magnitud y dirección a las reportadas en el estudio inicial de validación y en otros posteriores (6). Además, según el análisis factorial exploratorio, se verificó la validez del constructo al determinar que en su estructura interna los ítems se constituyeron en un solo factor que explicaría el $59,7 \%$ de la variabilidad total del fenómeno estudiado (gravedad de la enfermedad). También su coherencia interna demostró ser adecuada y similar a la previamente reportada $(6,7)$.

Con base en estos resultados y los de estudios previos $(6,7)$, el COPDSS puede utilizarse como una herramienta de clasificación de la gravedad de la EPOC muy valiosa, principalmente en zonas rurales o de difícil acceso, pues permite una fácil y rápida clasificación del paciente en los servicios de atención primaria. Este método no remplaza la espirometría, aunque es una útil herramienta en zonas donde no se dispone de esta prueba. Además, en España, por ejemplo, se evidenció que en la práctica clínica no se utilizaba regularmente la espirometría hasta en el $41 \%$ de los encuestados (7).

Por otra parte, el COPSS puede emplearse conjuntamente con otros cuestionarios para ayudar en el diagnóstico y seguimiento de los pacientes con EPOC (7). Se recomienda utilizarlo cada seis meses como parte 
de los programas de manejo de la EPOC para evaluar la evolución de la enfermedad de una forma más integral, además, podría ser útil en la investigación clínica como indicador de otro resultado importante por analizar en esta población.

En cuanto a la población estudiada, el 52 \% correspondía al sexo femenino, es decir que hubo una relación de 1:1 entre los sexos, similar a la del estudio de validación original desarrollado por Eisner, et al. (6), en tanto que, en la validación previa en España (7), predominó el sexo masculino (92\%) con una relación de 1:10. La edad promedio de los pacientes de este estudio fue un poco mayor a la de los pacientes del estudio original y a los de la validación en España $(6,7)$. A pesar de estas diferencias, y dado que la edad y el sexo no se han asociado con la gravedad de la EPOC, la validez del instrumento es adecuada.

Es importante destacar, como lo hacen diferentes autores (9-11), que la validación de un instrumento es un proceso que se nutre de diferentes perspectivas y no un componente de "todo o nada". En este sentido, esta es una primera versión de validación del cuestionario; específicamente en el contexto de la validez concurrente, es necesario aportar evidencias a partir de otras formas de validación que aporten al proceso y a la consolidación de una medida más confiable en nuestra población que evalúe la validez predictiva y la de constructo bajo la modalidad de grupos extremos que den fuerza a las inferencias basadas en los resultados del cuestionario.

\section{Agradecimientos}

Al Hospital de Santa Mónica (Dosquebradas, Risaralda), por su colaboración en la realización de este estudio.

\section{Referencias}

1. Burney PG, Patel J, Newson R, Minelli C, Naghavi M. Global and regional trends in COPD mortality, 1990-2010. Eur Respir J. 2015;45:1239-47. https://doi.org/10.1183/09031936.00142414

2. Gershon AS, Warner L, Cascagnette P, Victor JC, To T. Lifetime risk of developing chronic obstructive pulmonary disease: A longitudinal population study. Lancet. 2011;378:991-6. https://doi.org/10.1016/S0140-6736(11)60990-2

3. Caballero A, Torres-Duque CA, Jaramillo C, Bolívar F, Sanabria F, Osorio P, et al. Prevalence of COPD in five Colombian cities situated at low, medium, and high altitude (PREPOCOL study). Chest. 2008;133:343-9. https://doi.org/10.1378/chest.07-1361

4. Lozano R, Naghavi M, Foreman K, Lim S, Shibuya K, Aboyans V, et al. Global and regional mortality from 235 causes of death for 20 age groups in 1990 and 2010: A systematic analysis for the Global Burden of Disease Study 2010. Lancet. 2012;380:2095-128. https:// doi.org/10.1016/S0140-6736(12)61728-0

5. Yawn B, Mannino D, Littlejohn T, Ruoff G, Emmett A, Raphiou I, et al. Prevalence of COPD among symptomatic patients in a primary care setting. Curr Med Res Opin. 2009;25:2671-7. https://doi.org/10.1185/03007990903241350

6. Eisner MD, Trupin L, Katz PP, Yelin EH, Earnest G, Balmes J, et al. Development and validation of a survey-based COPD Severity Score. Chest. 2005;127:1890-7. https://doi.org/10.1378/chest.127.6.1890

7. Miravitlles M, Llor C, de Castellar R, Izquierdo I, Baró E, Donado E. Validation of the COPD severity score for its use in primary care. The NEREA study. Eur Respir J. 2009;33:519-27. https://doi.org/10.1183/09031936.00087208

8. Miravitlles M, Izquierdo I, Herrejón A, Torres JV, Baro E, Borja J. COPD severity score as a predictor of failure in exacerbations of COPD. The ESFERA study. Respir Med. 2011;105:7407. https://doi.org/10.1016/j.rmed.2010.12.020

9. Hulley SB, Cummings SR, Browner WS, Grady DG, Newman TB. Designing clinical research. Philadelphia: Lippincott Williams \& Wilkins; 2007. p. 351. 
10. Arias MR, Lloreda MJ, Lloreda MV. Psicometría. Madrid: Alianza Editorial, S.A.; 2006;430-5.

11. Streiner DL, Norman GR. Health measurement scales: A practical guide to their development and use. Oxford: Oxford University Press; 2008.

12. Vogelmeier CF, Criner GJ, Martínez FJ, Anzueto A, Barnes PJ, Bourbeau J, et al. Global strategy for the diagnosis, management, and prevention of chronic obstructive lung disease 2017 Report: GOLD Executive Summary. Arch Bronconeumol. 2017;53:128-49. https://doi.org/10.1016/j.arbres.2017.02.001

13. Miller MR, Hankinson J, Brusasco V, Burgos F, Casaburi R, Coates A, et al. Standardisation of spirometry. Eur Respir J. 2005;26:319-38. https://doi.org/10.1183/09031936.05.00034805

14. Guyatt GH, Townsend M, Berman LB, Pugsley SO. Quality of life in patients with chronic airflow limitation. Br J Dis Chest. 1987;81:45-54. https://doi.org/10.1016/0007-0971(87)90107-0

15. Estrada-Álvarez JM, Ossa-García X, del Quijano-del Gordo Cl, Bustos L, Urina DP, Pérez $\mathrm{CF}$, et al. Validation of the chronic respiratory questionnaire in the Colombian population with chronic obstructive pulmonary disease. Qual Life Res. 2015;24:2039-44. https://doi.org/10.1007/s11136-015-0939-1

16. González-García M, Torres-Duque CA, Bustos A, Jaramillo C, Maldonado D. Bronchial hyperresponsiveness in women with chronic obstructive pulmonary disease related to wood smoke. Int J Chron Obstruct Pulmon Dis. 2012;7:367-73. https://doi.org/10.2147/COPD.S30410

17. Alvis-Guzmán N, De la Hoz-Restrepo F, Montes-Farah J, Paternina-Caicedo A. Effect of biomass smoke on chronic obstructive pulmonary disease in rural localities of Colombia: $\mathrm{A}$ cross-sectional study. Rev Salud Pública (Bogotá). 2013;15:589-600.

18. Rodríguez DA, Kortianou EA, Alison JA, Casas A, Giavedoni S, Barberan-García A, et al. Heart rate recovery after 6-min walking test predicts acute exacerbation in COPD. Lung. 2017; 195:463-7. https://doi.org/10.1007/s00408-017-0027-0

19. Kuzmar I, Giraldo-Ospina CE, Acevedo-Osorio GO, Rua-Salas G. Morbilidad de la enfermedad pulmonar obstructiva crónica en Colombia. Resultados del estudio SANEPOC-22. Revista de la Facultad de Ciencias Médicas de Córdoba. 2018;75:19-24. https://doi.org/10.31053/1853.0605.v75.n1.16617

20. Barnes PJ, Burney PG, Silverman EK, Celli BR, Vestbo J, Wedzicha JA, et al. Chronic obstructive pulmonary disease. Nat Rev Dis Primers. 2015;1:15076. https://doi.org/10.1038/nrdp.2015.76

21. Celli B, Tetzlaff K, Criner G, Polkey MI, Sciurba F, Casaburi R, et al. The 6- minute-walk distance test as a chronic obstructive pulmonary disease stratification tool. Insights from the COPD Biomarker Qualification Consortium. Am J Respir Crit Care Med. 2016;194:1483-93. https://doi.org/10.1164/rccm.201508-16530C

22. Celli BR, Cote CG, Marín JM, Casanova C, Montes de Oca M, Méndez RA, et al. The bodymass index, airflow obstruction, dyspnea, and exercise capacity index in chronic obstructive pulmonary disease. N Engl J Med. 2004;350:1005-12. https://doi.org/10.1056/NEJMoa021322

23. Croft JB, Wheaton AG, Liu Y, Xu F, Lu H, Matthews KA, et al. Urban-rural county and state differences in chronic obstructive pulmonary disease - United States, 2015. MMWR Morb Mortal Wkly Rep. 2018;67:205-11. https://doi.org/10.15585/mmwr.mm6707a1

24. Banda HT, Thomson R, Mortimer K, Bello GAF, Mbera GB, Malmborg R, et al. Community prevalence of chronic respiratory symptoms in rural Malawi: Implications for policy. PLoS ONE. 2017;12:e0188437. https://doi.org/10.1371/journal.pone.0188437

25. Pothirat C, Chaiwong W, Phetsuk N, Pisalthanapuna S, Chetsadaphan N, Inchai J. A comparative study of COPD burden between urban vs rural communities in northern Thailand. Int J Chron Obstruct Pulmon Dis. 2015;10:1035-42.

https://doi.org/10.2147/COPD.S82303 